\title{
Surface Response Model for Quasielastic Scattering
}

\author{
Henning Esbensen
}

Physics Div., Argonne National Laboratory, Argonne, IL 60439-4843

\begin{abstract}
A review of the the surface response model and its applications to quasielastic nucleon-nucleus scattering at intermediate energies. CONF-8708102--9

INTRODUCTION

DE88 002976

The description of nucleon-nucleus inelastic scattering in terms of single-scattering has been very successful at intermediate energies. Nuclear structure is the most dominant feature at low excitations and forward scattering, and the Distorted Wave Impulse Approximation (DWIA) has been the most useful technique to extract structure information. A detailed discussion of the theoretical treatment of nucleon-nucleus scattering, specifically for charge exchange reactions, can be found in Refs. 1 and 2 . At larger scattering angles, the inelastic cross section develops into a broad quasielastic peak at higher excitations, and the signature of shell structure disappesrs. Moreover, the position and the width of this continuum peak is essentially insensitive to the choice of nuclear target.

The conventional DWIA has also been applied to quasielastic scattering (see for example Ref. 3). However, this method is very time-consuming at large scattering angles, since many different excitations of different multipolarities contribute to the inelastic cross section. It has therefore been useful to develop an approximate treatment that contains the main physics of quasielastic scattering.

In the following I will try to establish the connection between the DWIA and the much simpler Surface Response Model (Refs. 4, 5). I will give a short description of the Random Phase Approximation (RPA) that is used to calculate the nuclear response, and illusirate the spin-isospin dependence of the nucleon-nucleon t-matrix interaction, which is used to generate the excitations of the target nucleus. Finally, some of the applications of the surface response model to $\left(p, p^{\prime}\right),(p, n)$ and $\left({ }^{3} \mathrm{He}, t\right)$ reactions are reviewed.
\end{abstract}

\section{SINGLE SCATTERING CROSS SECTION}

The basic expression for the inelastic cross section in the DWIA is

$$
\frac{d^{2} \sigma}{d n d E}=\sum_{n} \frac{k_{n}}{k_{0}}\left(\frac{\mu}{2 \pi h^{2}}\right)^{2} \mid\left\langle\left.\psi_{n} \phi_{p}\left|V_{p t}\right| \psi_{0} \phi_{p}\right|^{2} \delta\left(\Delta E-\epsilon_{n O}\right)\right. \text {. }
$$

\section{MASTER}


There are three important ingredients in numerical DWIA calculations: 1. The diagonal interaction between projectile and target. The incoming and the scattered projectile waves $\phi_{p}$ and $p$, are calculated using an optical potential that fits elastic scatering.

2. The off-diagonal interaction $V_{p t}$ that generates the inelasic excitation. At intermediate energies the $t$-matrix interaction between free nucleons is often used. Medium corrections to the t-matrix are expected to be small at $300 \mathrm{MeV}$ and above. Effective, local interactions that fit the free t-matrix, when evaluated between plane waves, have also been constructed [Ref. 6].

3. The target wave functions $f_{n}$ for excited target states. They are calculated in the Random Phase Approximation from one-particle onehole excitations of the target.

At large beam energies one can use the Eikonal approximation to estimate the effect of the optical potential. The imaginary part of this potential $W(r)$ leads to an attenuation of the elastic beam. The real part is less important at high energies. The projectile transition density is then

$$
\delta \rho_{p}=\phi_{p}^{*}, \phi_{p}=\exp (i q) \exp \left(-\int d z W(r) / / v_{0}\right),
$$

where $q$ is the momentum transfer and the atter-uation depends on the path through the target. Furthermore, using the local, momentumdependent t-matrix interaction, $v_{p t}=t(q) \delta\left(r_{p}-r_{t}\right)$, Eq. (1) takes the form

$$
\frac{d^{2} \sigma}{d D d E}=\sum_{n} \frac{k_{n}}{k_{0}}\left(\frac{\mu}{2 \pi k^{2}}\right)^{2}|t(q)|^{2}\left|\left\langle\psi_{n}\left|\delta p_{p}\right| \psi_{0}\right\rangle\right|^{2} \delta\left(\Delta E-\epsilon_{n}\right) .
$$

It is convenient to extract an overail normalization factor that depends on the target density $\rho_{A}(r)$ and the absorption,

$$
N_{\text {eff }}=\left\langle\left. 0|| \delta \rho_{p}\right|^{2} \mid 0\right\rangle=\int \mathrm{d} r \rho_{\Lambda}(r) \exp \left(-2 \int \mathrm{dz} W(r) / / \mathrm{v}_{0}\right) .
$$

It represents the effective number of target nucleors that contribute to single scattering. We can also define $z$ response function by

$$
S(q, \Delta \mathrm{B})=\sum_{\mathrm{n}}\left|\left\langle\left.\psi_{\mathrm{n}}|| \delta \rho_{\mathrm{p}}\right|^{2} \mid \psi_{0}\right\rangle\right|^{2} \delta\left(\Delta \mathrm{E}-\epsilon_{\mathrm{n} 0}\right) / \mathrm{N}_{\mathrm{eff}} .
$$

The total response can be obtained by closure if we neglect the effects of RPA correlations and Pauli blocking. Under these assumptions the normalization has been chosen such that the total response is one. With these definitions the inelastic cross section is

$$
\frac{d^{2} \sigma}{d n d E}=\frac{k_{n}}{k_{0}} \cdot \frac{d \sigma_{N N}^{e \ell}}{d n} \cdot N_{e f f}=S(q, \Delta E) .
$$


The square of the t-matrix has here been replaced by the elastic nucleon-nucleon cross section. This expression is in fact the basic formula in the surface response model.

An important feature of nucleon-nucleus scattering is the strong absorption in the nuclear medium. In the surface response model we use Glauber theory for single-scattering, which is equivalent to making the following substitution for the optical potential

$$
2 W(r) / h_{0}+\rho_{\Lambda}(r) \sigma_{N N} \text {, }
$$

where $\sigma_{N N}$ is the total nucleon-nucleon cross section. Typically $\sigma_{N N}$ $\sim 30 \mathrm{mb}$, and the mean free path is of the order of $2 \mathrm{fm}$ at nuclear matter density. The quasielastic (single-scattering) cross section therefore originates from a narrow ring around the nucleus (when the scattering angle is not too large).

A major simplification is made by replacing the response of finite nuclei by the surface response of semi-infinite nuclear matter. The numerical calculation of the latter response is much faster and it contrins the RPA dynamics of a nuclear surface. It does not contain the shell structure of finite nuclei, but this aspect does not seem to be very crucial for the observed quasielastic peaks.

\section{LINEAR RESPONSE THEORY (RPA)}

The calculation of the response is based on mean-field theory for the single-particle excitations and the effects of residual interactions in the medium are included by solving the RPA equation. The fundamental operator in this framework is the so-called retarded polarization operator. For independent particles it is defined by

$$
\begin{aligned}
& \Pi_{0}\left(r, r^{\prime}, \Delta E\right)=\frac{-4}{(2 \pi)^{3}} \int_{\epsilon_{i}<\epsilon_{p}} d k_{i} \phi_{i}^{*}(r)\left[\left(H_{0}-\epsilon_{i}-\Delta E-i \eta\right)^{-1}\right. \\
& \left.+\left(B_{0}-\epsilon_{i}+\Delta E+i \eta\right)^{-1}\right] \phi_{i}\left(r^{\prime}\right),
\end{aligned}
$$

where the sum is over all occupied single-particle states below the Fermi surface and $H_{0}$ is the single-particle Hamiltonian. The asscciated response to an external field $F(r) \exp (-i \Delta E t / \not)$ that acts on the system is

$$
S_{0}(\Delta E)=-\frac{1}{\pi} \operatorname{Im} \int d r d r^{\prime} F^{*}(r) \Pi_{0}\left(r, r^{\prime}, \Delta E\right) F\left(r^{\prime}\right) .
$$

If we insert a complete set of final states into Eq. (8) the independent particle response is 


$$
\begin{aligned}
S_{0}(\Delta \mathrm{E})= & \sum_{\epsilon_{\mathrm{i}}\left\langle\epsilon_{\mathrm{F}}\right.}\left|\left\langle\phi_{\mathrm{f}}|\mathrm{F}(\mathbf{r})| \phi_{\mathrm{i}}\right\rangle\right|^{2} \delta\left(\Delta \overline{\mathrm{E}}-\dot{\epsilon}_{\mathrm{fi}}\right), \\
& \left.\epsilon_{\mathrm{f}}\right\rangle \epsilon_{\mathrm{F}}
\end{aligned}
$$

which is the expression one would expect in first order perturbation theory. The polarization operator furthermore determines the density induced by the external field,

$$
\delta \rho(\mathbf{r}, \Delta E)=j \mathrm{~d} \mathbf{r}^{\prime} \Pi_{0}\left(\mathbf{r}, \mathbf{r}^{\prime}, \Delta E\right) F\left(\mathbf{r}^{\prime}\right) .
$$

A residual interaction $V_{\text {res }}\left(r, r^{\prime}\right)$ between the particles in the medium will modify the response, since the induced density will generate an induced potential, which is

$$
\delta V(r, \Delta E)=\int d r^{\prime} V_{r e s}\left(r, r^{\prime}\right) \delta \rho(r, \Delta E) .
$$

With these definitions we can now formulate the RPA equation as a self-consistent linear equation for the induced density

$$
\begin{aligned}
& \delta \rho(r, \Delta E)=\int d r^{\prime} \Pi_{0}\left(r, r^{\prime}, \Delta E\right)\left\{F\left(r^{\prime}\right)\right. \\
& \left.+\int d r^{\prime \prime} v_{r e s}\left(r^{\prime}, r^{\prime \prime}\right) \delta \rho\left(r^{\prime \prime}, \Delta E\right)\right\} .
\end{aligned}
$$

The solution to this integral equation,

$$
\delta \rho(\mathbf{r}, \Delta \mathrm{E})=\int \mathrm{d} \mathbf{r}^{\prime} \mathrm{I}_{\mathrm{RP}_{\mathrm{\Lambda}}}\left(\mathbf{r}, \mathbf{r}^{\prime}, \mathrm{AE}\right) \mathrm{F}\left(\mathbf{r}^{\prime}\right),
$$

determines the RPA polarization operator which we can write (using a compact operator rotation)

$$
\mathrm{I}_{\mathrm{RPA}}=\left(1-\mathrm{I}_{0} \mathrm{v}_{\mathrm{res}}\right)^{-1} \Pi_{0} \text {. }
$$

The RPA response is obtained by inserting $\Pi_{R P A}$ into Eq. (9). There is no simple, closed expression for the total $\mathrm{RPA}_{\mathrm{A}}$ response, integrated over all excitation energies at a fixed momentum transfer. It is affected by the residual interacticn and Pauli blocking. The total energy weighted response, however, obeys a simple sum rule and it is identical to the energy weighted sum of the free response if the residual interaction commutes with the external field.

The above formulation of the RPA is quite general. However, solving the integral equation for the RPA polarization operator can in general be very time-consuming. It is therefore useful to study the response of systems with simple symmetries. The simplest system is infinite nuclear matter, where the the RPA equation can be reduced to 
an algebraic equation in momentum space. A discussion for spherical nuclei, based on Hartree-Fock theory and Skyrme interactions, can be found in Ref. 7.

For semi-infinite nuclear matter one can exploit the translational invariance along the surface. Thus the momentum transfer $\mathbf{K}$ parallel to the surface becomes a simple parameter in the RPA equation, and the numerical problem is reduced to calculating the non-local polarization operator $\Pi\left(s, s^{\prime}, K, \Delta E\right)$ for the $s$-direction perpendicular to the surface. This is discussed in detail in Ref. 4. The field that generates the surface response of semi-infinite nuclei, used to simulate the surface response of finite nuclei, is determined by the absorption inside the nucleus. It is similar to the projectile transition density (2) and it has the form $F(s) \exp (i(q r-\Delta E t / \not)$. The prescription for constructing this field is described in Sect. IIB of Ref. 8.

The residual interactions used in the calculation of the RPA responses are given in Ref. 4. The isoscalar interaction is determined by the self-consistency of the mean field. The empirical spin and the isovector interactions have been extracted from the spectroscopy of discrete transitions in finite nusisi. An important question is how well these interactions apply to the response of nuclei at the large momentum transfers associated with quasielastic scattering.

THE NUCLEON-NUCLEON t-MATRIX INTERACTION

The t-matrix interaction can be separated into the different components, which generate the different isospin-spin (T,S) excitations of the target nucleus. The calculation of these components from the empirically known proton-proton and proton-neutron phase-shifts is described in detail in sect. 2.1 of Ref. 2. The inelastic cross section for each $(T, S)$ excitation channel is given by an expression that is similar to Eq. (6),

$$
\left(\frac{\mathrm{d}^{2} \sigma}{\mathrm{d} 0 \mathrm{dE}}\right)_{\mathrm{T}, \mathrm{S}}=\frac{\mathrm{k}_{\mathrm{n}}}{\mathrm{k}_{0}}\left(\frac{\mathrm{d} \sigma_{\mathrm{NN}}^{\mathrm{\ell}}}{\mathrm{dn}}\right)_{\mathrm{T}, \mathrm{S}} \cdot \mathrm{N}_{\mathrm{eff}} \cdot \mathrm{S}_{\mathrm{T}, \mathrm{S}}(\mathrm{q}, \Delta \mathrm{E})
$$

where the RPA response depends on the spin and isospin of the excitation via the residual interaction. The inclusive ( $\left.p, p^{\prime}\right)$ cross section is given by the sum over all $(\mathrm{T}, \mathrm{S})$ channels.

One can emphasize different parts of the nuclear response by choosing specific reactions. In charge exchange reactions, for example, the isovector response is selected. In experiments with polarized protons one can isolate the spin response of nuclei.

The energy dependence of the t-matrix interaction at zero degrees scattering is shown in Fig. 1. It clearly shows that the isoscalar response is the dominant contribution in inclusive $\left(p, p^{\prime}\right)$ reactions at 
forward scattering. In $(p, n)$ reactions, on the other hand, the coupling to the isovector spin excitation channel is large. This has made it possible to study the giant Gamow-Teller resonance.

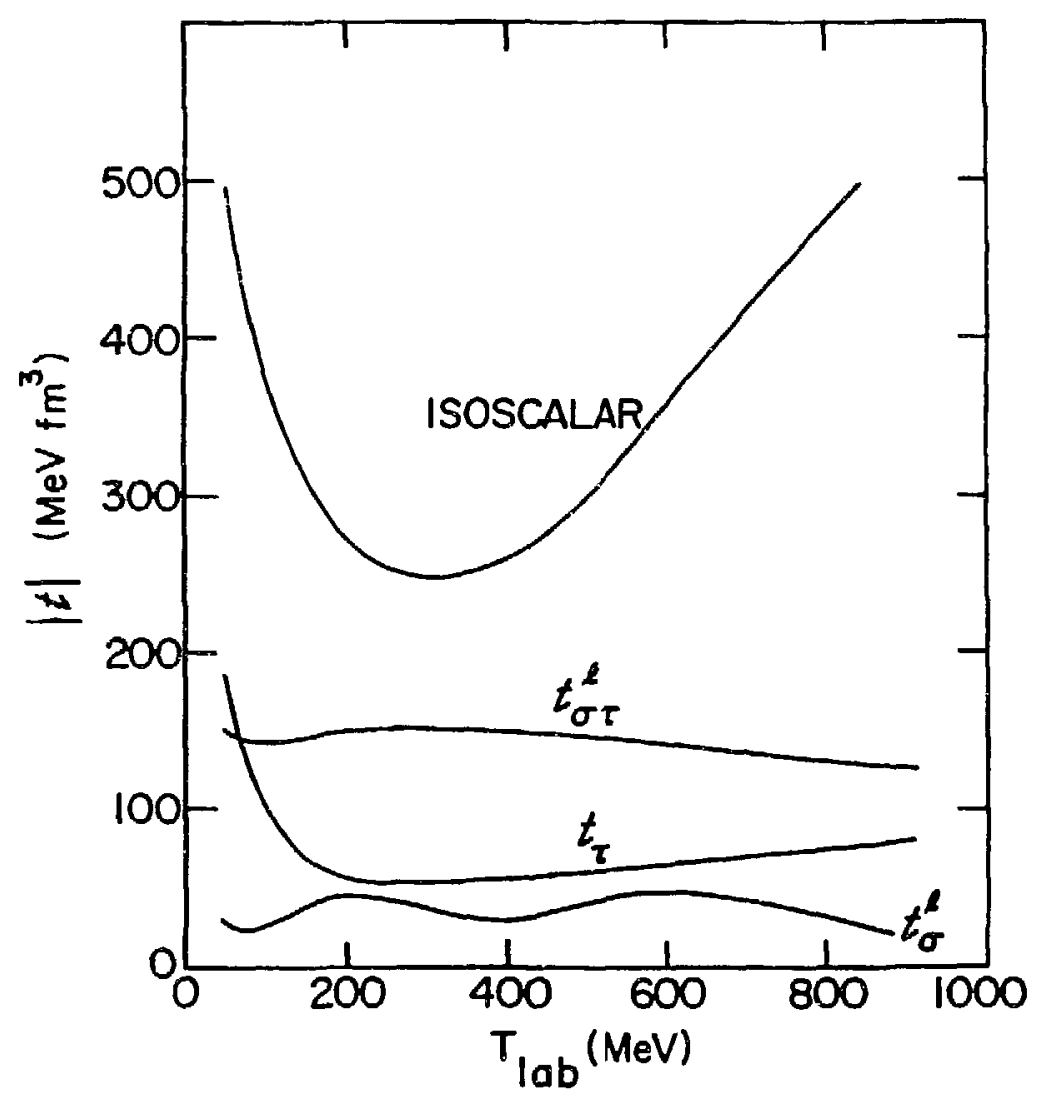

Figure 1

Energy dependence of different spin-isospin components of the t-matrix interaction for nucleon-nucleon scattering at zero degrees.

The dependence of the t-matrix interaction on the momentum transfer is illustrated in Fig. 2 at a beam energy of $300 \mathrm{MeV}$. At large momentum transfers, the coupling to spin excitations becomes more important. This figure also shows a detailed decomposition of the interaction that leads to spin-excitations of the target, viz. 


$$
\begin{aligned}
t_{S=1} & =t^{S 0} \sigma_{2 N}+t^{\ell}\left(\sigma_{1} \cdot \hat{q}\right)\left(\sigma_{2} \cdot \hat{q}\right)+t^{\rho} \frac{1}{\sqrt{2}}\left(\sigma_{1} \times \hat{q}\right) \cdot\left(\sigma_{2} \times \hat{q}\right) \\
& +t^{u} \frac{1}{\sqrt{2}}\left(\sigma_{1 N} \sigma_{2 N}-\sigma_{1 P} \sigma_{2 P}\right)
\end{aligned}
$$

where $\sigma_{1}$ and $\sigma_{2}$ are Pauli matrices for the projectile and target, respectively. Here the $\hat{q}$ direction is along the momentym transfer, $P$ is along the average initial and final momentum, and $N=q \times P$ is perpendicular to the scattering plane.

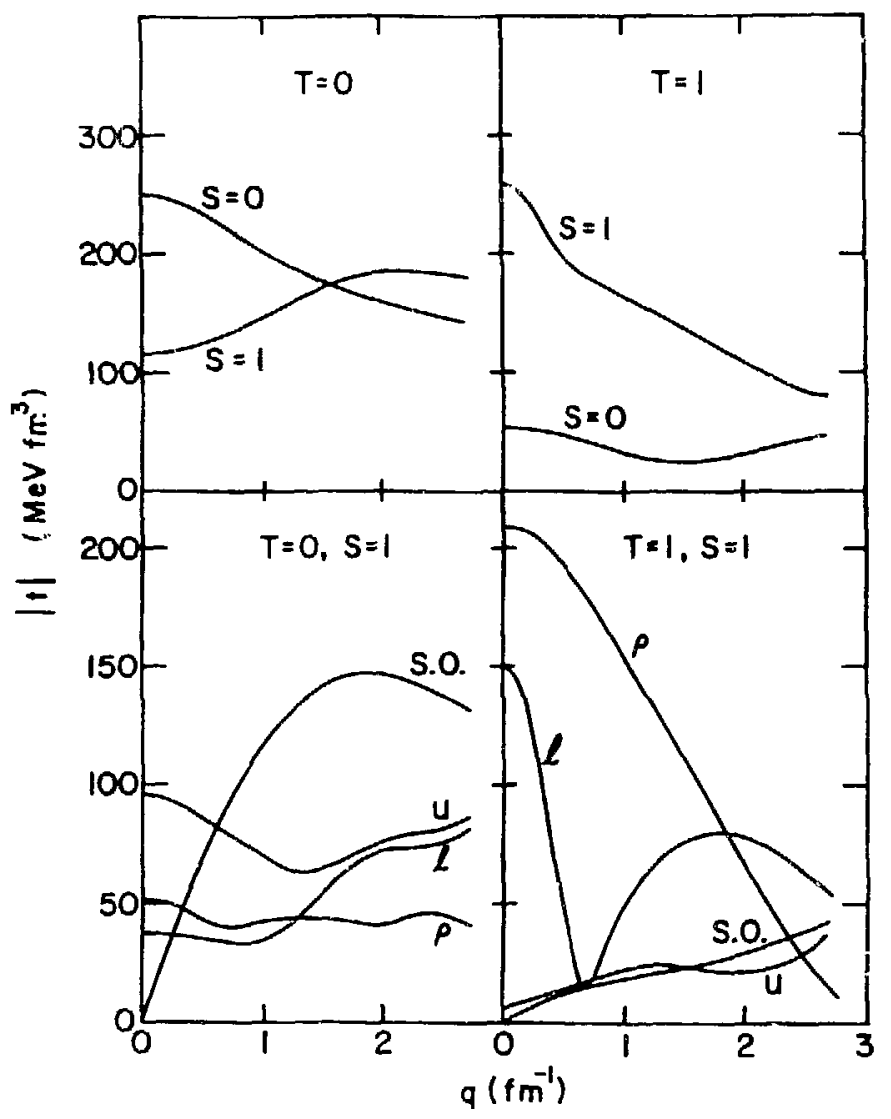

Figure 2

Nucleon-nucleon t-matrix interactions at $300 \mathrm{MeV}$ as functions of the momentum transfer. The spin-isospin representation is associated with target excitations. The different components of the spin excitation amplitudes shown in the lower two figures are defined in Eq. (17). 
The spin-orbit term $\left(\mathrm{t}^{\mathrm{SO}}\right)$ is seen to dominate the isoscalar spinexcitation at large momentum transfers. In the isovector channel $(\mathrm{T}=1)$, the longitudinal interaction, $\mathrm{t}^{\ell}$, is associated with pion-exchange, whereas $t^{\rho}$ has the same spin-dependence as rho-meson exchange. The spin-orbit term is much weaker. The term $t^{\mathrm{u}}$ is also weak, and it has a more subtle origin, from non-local interactions and exchange. A discussion of the nucleon-nucleon t-matrix in terms of effective interactions can be found in Sect. III of Ref. 1.

The spin response of nuclei has been studied in experiments with polarized protons, and the spin-flip probability has been measured as a function of excitation energy at different scattering angles. The more delicate separation of the spin response into a longitudinal and a transverse component has also been studied experimentally.

\section{APPLICATIONS TO (p,p') REACTIONS}

The Surface Response Model has been applied to a variety of nucleon-nucleus scattering reactions. A systematic comparison to inclusive ( $\left.p, p^{\prime}\right)$ spectra can be found in Ref. 5 . The position and the width of the quasielastic peak is quite well described by the model. The target dependence of the quasielastic peak is mainly contained in the normalization factor $\mathrm{N}_{\text {eff' }}$ which is calculated using Glauber theory for single-scattering. The agreement with measured peak heights, both for ${ }^{12} \mathrm{C},{ }^{58} \mathrm{Ni}$ and ${ }^{208} \mathrm{~Pb}$, is a success of the Glauber calculation. It confirms the picture that quasielastic scattering is a surface reaction. Alternatively, assuming that the Glauber calculation is valid, then the agreement with the measured spectra shows that the quasielastic response of nuclei is insensitive to the size of the target nucleus, and that it is quite well represented by the surface response of semi-infinite nuclear matter. The largest discrepancy with the data is observed in the high energy tail of the energy loss spectrum. It has been associated with multi-step processes, and an estimate of double scattering can be found in Ref. 11.

There is a medium correction to the Glauber calculation that one can easily account for. It concerns the total nucleon-nucleon cross section, which is reduced compared to the value for free scattering, mainly due to Pauli blocking. A consistent way of calculating it within the model is described in Ref. 4, and accounting for it improves the comparison to the data.

The inclusive ( $\left.p, p^{\prime}\right)$ spectra are insensitive to the effect of the residual interactions on the $R P A$ responses at large momentum transfers. This is a consequence of a cancellation between different contributions. The isoscalar response is enhanced at lower excitations but the responses in the other spin-isospin channels are reduced, due to their repulsive residual interactions. The situation is reversed at excitations above the quasielastic peak. Some effect of the attractive isoscalar interaction can be seen at forward scattering, where the 
isoscalar channel starts to dominate and enhances the inclusive spectrum (see f.ex. Fig. 8 in Ref. 4).

The sensitivity to residual interactions can be increased by gating on more exclusive reactions. An example is the spin-flip probabilities reported in Ref. 9. They show a dramatic deviation from the value for free scattering. The surface response model can explain the qualitative trend of the data; namely, a reduction at low excitation energies due to the enhancet isoscalar response in the inclusive cross section and a reduced responze in the spin-flip channels. The model also predicts some enhancement of the spin-flip probability ar excitation energies above the quasielastic peak but it is not as large as the measured values. There is fresently no explanation for this discrepancy.

\section{APPLICATIONS TO (D,n) REACTIONS}

The existing data on quasielastic $(p, n)$ reactions are not as extensive as the ( $\left.p, p^{\prime}\right)$ data at intermediate energies. Systematic measurements have been performed at $200 \mathrm{MeV}$ (Ref. 12). The surface response model underestimates the quasielastic peaks, although a small value of $\sigma_{\mathrm{NN}} \sim 25 \mathrm{mb}$ (consistent with the effective value corrected for Pauli blocking) was used in the Glauber calculation. The model predicts a significant contribution from double-scattering at the larger scattering angles. An example is given in Fig. 2 of Ref. 11 . The data have been reproduced by DWIA calculations, which employed an optical model with a weak absorption (see Ref. 3). The true absorption is probably somewhere between these two extremes, with some amount of double-scattering at the quasielasti: peak for larger scattering angles.

There are also some data at $800 \mathrm{MeV}$. Spectra at zero degrees scattering have been measured on a variety of nuclei, and the delta peak is clearly identified. 13,14 Spectra at finite scattering angles have been measured for reactions on lithium and carbon targets.

It would be very valuable to have more $(p, n)$ data at higher beam energies. The relative contribution from multistep processes at the quasielastic peak decreases with increasing beam energy (see Ref. 11), roughly like $1 / E$ for a fixed momentum transfer. Moreover, the choice of absorption is less controversial at higher energies. Such data could provide more direct informations about the isovector spin response of nuclei at large momentum transfers, and they would supplement the existing $\left(p, f^{\prime}\right)$ results of Refs. 9 and 10 , which unavoidably contain a background from the isoscalar spin response. 


\section{APPLICATIONS TO $\left({ }^{3} \mathrm{He}, \mathrm{t}\right)$ REACTIONS}

The surface response model can be generalized to describe $\left({ }^{3} \mathrm{He}, \mathrm{t}\right)$ reactions, which have been studied extensively at a beam energy of 2 $\mathrm{GeV}$. $^{15-17}$ The excitations in quasielastic scattering are assumed to be generated by the free nucleon-nucleon t-matrix interaction for charge exchange. The finite size of the projectile is accounted for by including the point-charge form factor $F_{f}\left(q_{\mu}^{2}\right)$ for ${ }^{3} \mathrm{He}$. The quasielastic cross section has a form similar to Eq. $(16)$,

$$
\frac{d^{2} \sigma}{d n d E}=C \times \sum_{S=0,1}\left|t_{T, S}(q)\right|^{2} \cdot N_{\text {eff }} \cdot S(q, \Delta E)\left|F_{c}\left(q^{2}-(w / c)^{2}\right)\right|^{2},
$$

where the factor $\mathrm{C}$ depends on the kinematics of the reaction.

The model can also be generalized to calculate the cross section for delta-excitations in the target. The $\left({ }^{3} \mathrm{He}, t\right)$ reaction is in a way much simpler than the corresponding $(p, n)$ reaction, since there is a broad background in the latter from the neutron decay of the delta. The delta-excitation is generated by the pion-exchange interaction $V_{N A, N N}$. The position and the width of the delta resonance can be included in the response function for the target nucleus. This is described in detail in Ref. 18. The model is adjusted to describe the measured ${ }^{15}$ delta-excitation on free protons. This determines an overall distortion factor that represents the scattering dynamics prior to the delta decay, and once fixed, it is assumed to be independent of excitation energy and scattering angle.

The model has been applied to delta-excitations induced by $\left({ }^{3} \mathrm{He}, \mathrm{t}\right)$ reactions at zero degrees scattering. ${ }^{18}$ In order to fit the peak position measured in reactions with heavy nuclei, ${ }^{16}$ which is shifted by about $50 \mathrm{MeV}$ compared to reactions on free protons, it was necessary to adjust the self-energy of the delta in a nuclear medium. Similar adjustments are made to fit pion-nucleus scattering.

The results of calculations, both for the quasielastic and the delta peak, are compared in Fig. 3 to the measured spectrum 17 at 5 degrees scattering on ${ }^{12} \mathrm{C}$. Both calculations employed a total ${ }^{3}$ He-nucleon cross section of $111 \mathrm{mb}$ in the Glauber calculation of $\mathrm{N}_{\text {eff }}$. The quality of the comparison to the two measured peaks is about the same. There is a serious discrepancy in the dip-region between the two peaks, and also in the tail at very large excitations. 


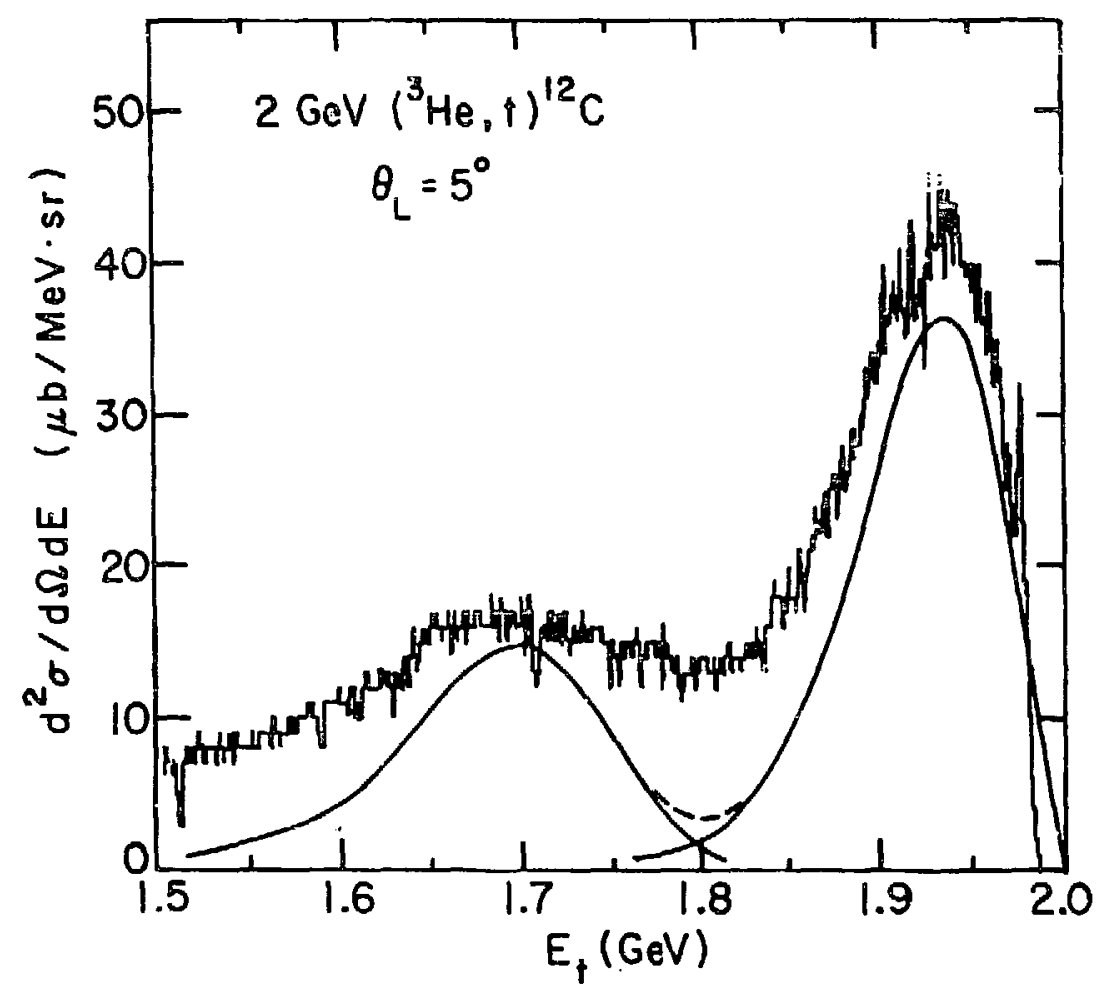

Figure 3

Energy spectrum for $2 \mathrm{GeV}\left({ }^{3} \mathrm{He}, \mathrm{t}\right)$ reactions on 12 at 5 degrees scattering. The data are from Ref. 17.

The magnitude of the cross sections are very sensitive to the choice of the form factor. An apparent discrepancy between the calculated and the measured peak heights at larger scattering angles can essentially be removed by replacing the square of the ${ }^{3} \mathrm{He}$ form factor by the correct product of the ${ }^{3} \mathrm{He}$ and the triton form factor. The position of the measured quasielastic peak, however, is still shifted by about 10 and $25 \mathrm{MeV}$ to lower excitations at 6 and 7 degrees, respectively. There is presently no explanation for this discrepuncy.

It will be important in future studies to make a simultaneous and consistent description of particle-hole and delta-hole excitations. In particular, it will be important to understand the delta dynamics in the nuclear medium, and to make a description that is consistent with pion scattering. 
We are presently working on a generalization of the surface response mode! to describe quasielastic pion scattering. The strong absorption of the pj- in the resonance region (see Fig. 4) implies that the pion scattering ' is place near the surface of the target nucleus. As a final illustration we show in Fig. 5 the differential pion-nucleon cross section associated with isoscalar excitations of a nucleus. The pion has a strong coupling to spin excitations at 60 to 100 degrees scattering. The corresponding cross sections for couplings to isovector excitationis are about a factor of four smaller than the isoscalar cross sectione due to the dominance of the coupling to the delta.

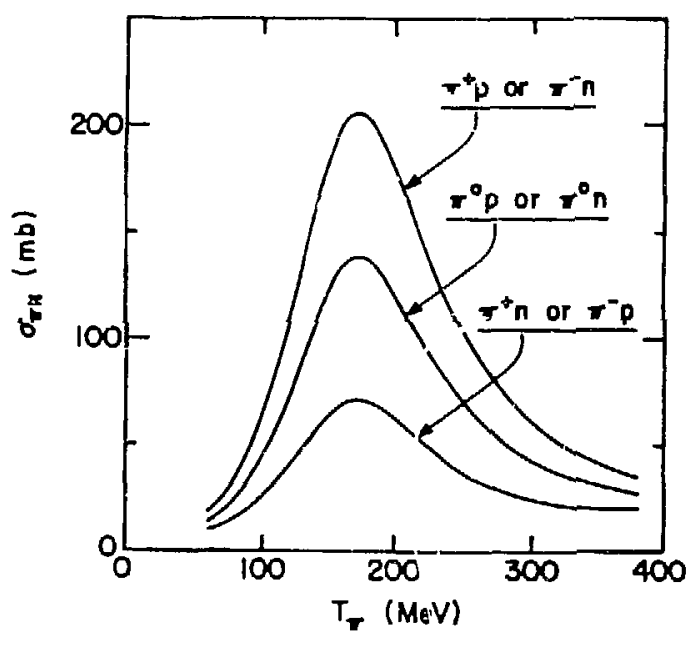

Figure 4

Energy dependence of the total $\pi \mathrm{N}$ cross section.

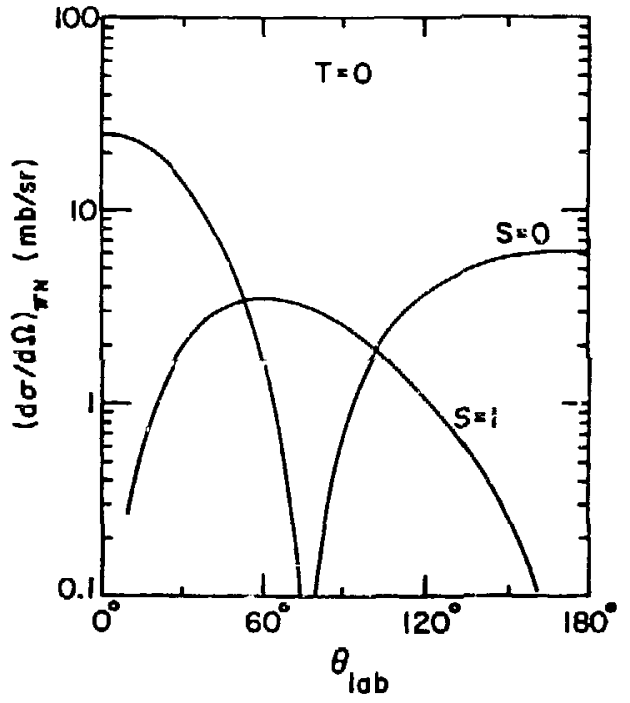

Figure 5

Differential $\pi \mathrm{N}$ cross sections for $200 \mathrm{MeV}$ pions. The components for isoscalar $(S=0)$ and isoscalar spin $(\mathrm{S}=1)$ excitations are shown.

This work supported by the U. S. Department of Energy, Nuclear Physics Division, under contract W-31-109-ENG-38. 
1. "Spin Excitations in Nuclei", eds. F. Petrovich et al., Plenum, NY (1984).

2. G. F. Bertsch and H. Esbensen, Rep. Prog. Phys. 50 (1987) 607.

3. F. Oste:feld, D. Cha \& J. Speth, Phys. Rev. C31 (1985) 372; M. Yabe, F. Osterfeld and D. Cha, Phys. Lett. B178 (1986) 5.

4. H. Esbensen and G. F. Bertsch, Ann. Phys. $157(1984) 255$.

5. H. Esbensen and G. F. Bertsch, Phys. Rev. C34 1986$) 1419$.

6. W. G. Love and M. A. Franey, Phys. Rev. C31 (1985) 488.

7. G. F. Bertssh and S. F. Tsai, Phys.Rep. 18 (1975) 125.

8. H. Esbensen, H. Toki and G. F. Bertsch, Phys. Rev. C31 (1985) 1816.

9. C. Glashausser et al., Phys. R.ev. Lett. 58 (1987) 2404.

10. J. M. Moss et al., Phys. Rev. Lett. 53 (1984) 144; Phys. Rev. C34 (1986) 627.

11. H. Esbensen and G. F. Bertsch, Phys. Rev. C32 (!985) 553.

12. C. Gaarde et al., Nucl. Phys. A369 (1981) 258.

13. B. E. Bonner et al., Phys. Rev. C 18 (1978) 1418.

14. R. G. Jeppesen, Thesis (University of Colorado, 1986).

15. C. Ellegaard et al., Phys. Lett. 154B (1985) 110.

16. D. Contardo et al., Phys. Lett. 168B (1986) 331.

17. I. Berggvirt et al., Nucl. Phys. A (in press).

18. H. Esbenser anú T.-S. H. Lee, Phys. Rev. C32 (1985) 1966.

19. H. Esbensen, R. Smith and E. Siciliano (work in progress) 